\title{
Irradiation des femmes enceintes travaillant sur terminal informatique à écran cathodique
}

\author{
G. WOLBER* \\ (Manuscrit reçu le 20 février 1985)
}

\begin{abstract}
RÉSUME
En raison du développement explosif de l'usage de terminaux informatiques à écran cathodique, on s'est interrogé sur l'importance de l'irradiation du personnel par les émissions parasites de rayons $X$ par ces matériels. On traite ici le cas des femmes enceintes, car il s'agit essentiellement de main-d'œuvre féminine. Dans les cas des plus défavorables, la dose reçue par le fœtus aux points les plus exposés est estimée à $0,01 \mu \mathrm{Sv}(1 \mu \mathrm{rem})$. Ce niveau est tellement bas qu'on ne peut lui attribuer une quelconque pathologie. II n'y a donc pas lieu d'écarter les femmes enceintes des travaux sur terminaux à écran.
\end{abstract}

\section{ABSTRACT}

Because of the rapid proliferation of the use of video display units for data processing, we have investigated the levels of the personnel exposure to parasit $X$-rays emission from these devices. The case of pregnant women was investigated because the greater part of the staff involved is female. In the worst case, the dose equivalent received by the fœtus at the most exposed point is estimated at $0,01 \mu \mathrm{Sv}$. This level is too low to cause any pathology. There is, therefore, no reason to discard pregnant women from the use of video display units.

Les applications de la bureautique et de la télématique se développent de façon explosive. La plupart de ces matériels, à quelques exceptions près, utilisent des consoles à écran cathodique. Ces postes de travail ont posé des problèmes ergonomiques dont celui des rayonnements $X$ parasites émis par les équipements. Une étude, parue l'an dernier dans cette revue [6], a montré que l'inquiétude provoquée par ce dernier problème n'était pas fondée. S'agissant le plus souvent d'une main-d'œuvre féminine, il est logique de s'interroger sur l'importance de l'irradiation des femmes enceintes affectées à ces travaux. Ce sujet n'avait pas été examiné dans l'étude précédente dont l'exposé qui suit est un complément. La plupart des développements sont supposés connus et ce qui a déjà été traité ne sera pas repris.

* EdF, Comité de radioprotection, 3, rue de Messine, 75384 Paris Cedex 08. 


\section{CONDITIONS D'IRRADIATION DES FEMMES ENCEINTES}

Les terminaux à écran peuvent se classer en 3 catégories selon le tube cathodique utilisé pour l'affichage : tube monochrome, tube graphique couleur à pénétration, tube trichrome. Le fonctionnement de ces dispositifs a été décrit dans l'article précédent.

Les deux premières catégories de matériels n'entraînent pas d'irradiation significative des opératrices: les niveaux sont estimés $10^{20}$ fois plus bas que l'irradiation naturelle qui varie elle-même, en France, dans le rapport de 1 à 3 sans qu'on en tienne compte dans le calcul de l'irradiation des personnes.

Nous étudierons donc essentiellement le cas des postes de traitement d'image couleur, les seuls utilisant les tubes cathodiques trichromes, donc susceptibles de délivrer des doses d'un niveau accessible à la mesure.

\subsection{Hypothèses de calcul des doses reçues par le fœtus}

Il s'agit d'évaluer l'irradiation de l'enfant au cours de la grossesse. Elle dépend du temps d'exposition, de la position de l'embryon et du fœtus dans l'utérus par rapport à l'axe du tube cathodique et de l'atténuation des rayons $X$ due à l'épaisseur de la paroi utérine et de l'abdomen.

L'horaire journalier maximal est de 6 heures. En supposant que la femme enceinte travaille jusqu'au début de ses congés de maternité, soit 7 mois à partir du début de la grossesse, on arrive à une durée totale maximale du travail devant écran de 900 heures.

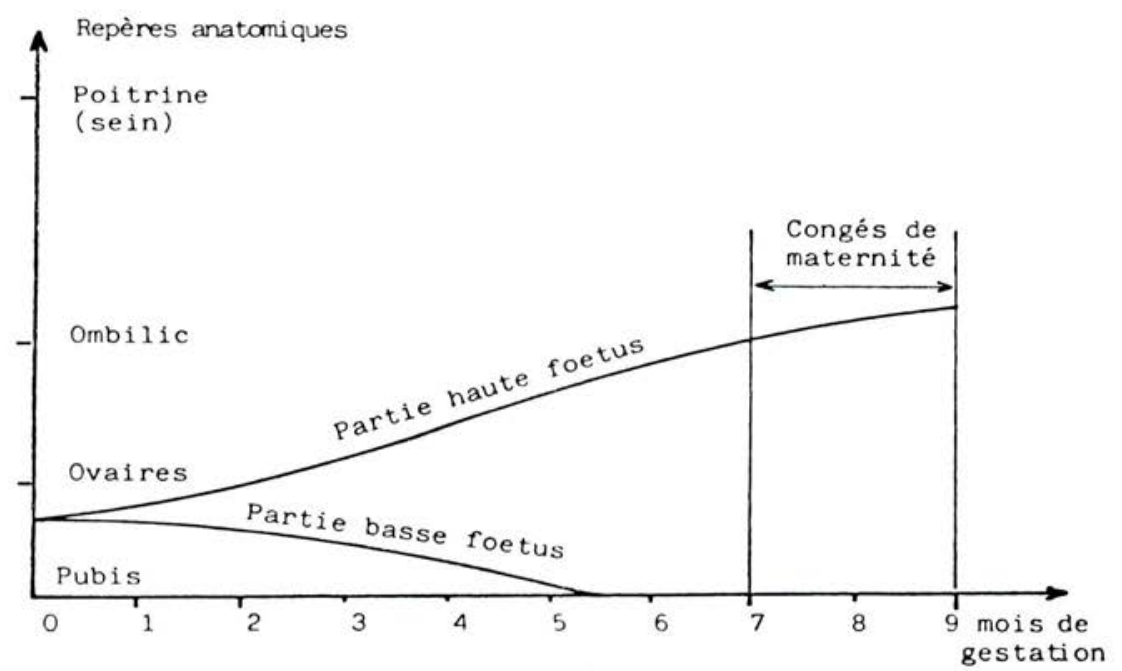

Fig. 1. - Variations au cours de la grossesse de la position du fœetus par rapport au repère anatomique de la mère. 
Pendant les 2 premiers mois, l'embryon se trouve situé légèrement en-dessous des ovaires, soit au moins à $64 \mathrm{~cm}$ de l'axe du tube cathodique (fig. 1 et 2) : il reçoit donc approximativement la même dose que les ovaires durant cette période [3]. Le développement du fœtus fait qu'au $4^{\text {e }}$ mois, il arrive à la hauteur de l'ombilic, c'est-à-dire à $55 \mathrm{~cm}$ au-dessous de l'axe du tube cathodique.

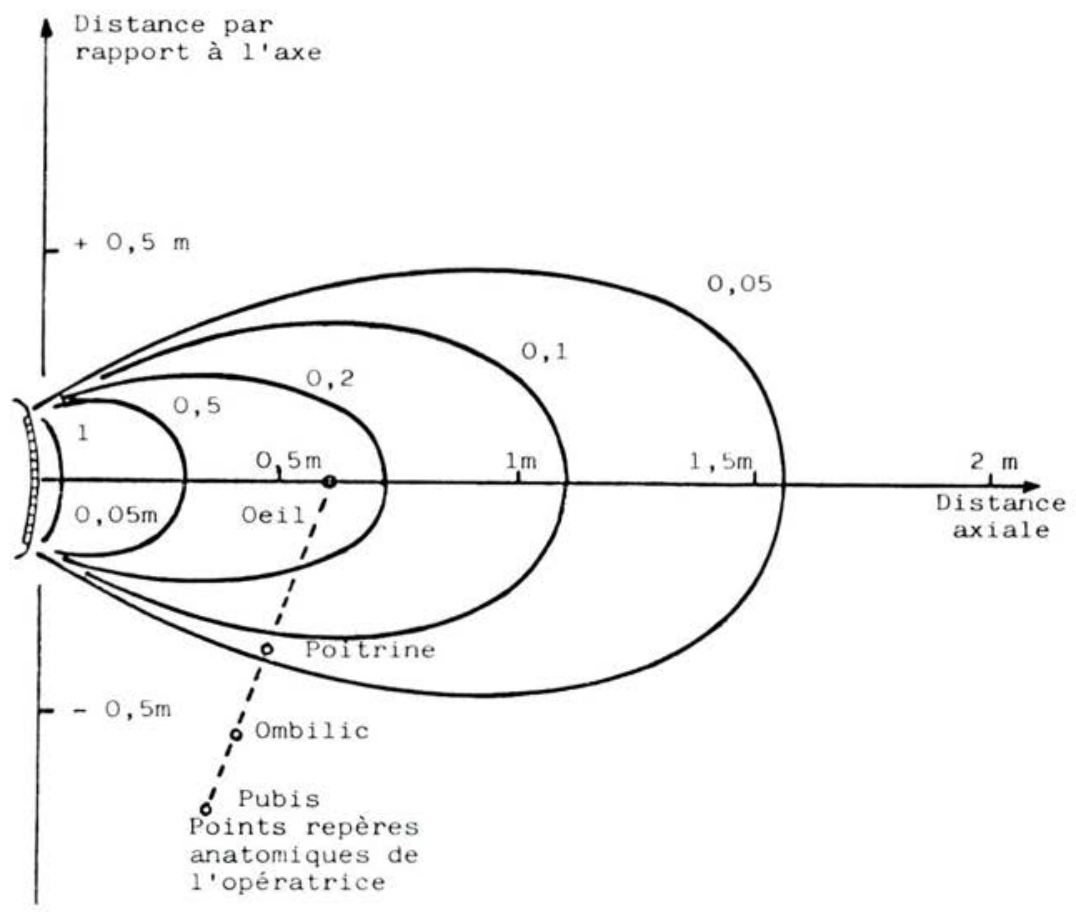

Fig. 2.- Courbes isodoses, dans le plan vertical, du rayonnement d' un tube cathodique polychrome de $47 \mathrm{~cm}$ de diagonale à face sphérique de $70 \mathrm{~cm}$ de rayon et alimenté a $25 \mathrm{kV}$.

Pendant la période fœtale, l'épaisseur de la paroi utérine varie entre 10 et $15 \mathrm{~mm}$. L'épaisseur totale de la paroi abdominale (épiderme + derme + hypoderme) est de l'ordre de $6 \mathrm{~mm}$ [2]. L'épaisseur moyenne qui sépare le fœtus de l'extérieur est donc de 20 mm.

\subsection{Facteurs de conversion de dose choisis}

Comme dans l'article précédent, on envisage le cas d'irradiation maximale avec un tube trichrome alimenté avec une tension de post-accélération de $25 \mathrm{kV}$. La figure 2 reproduit les courbes isodoses d'un de ces tubes de $47 \mathrm{~cm}$ de diagonale avec une face sphérique de $70 \mathrm{~cm}$ de rayon (chaque courbe isodose est exprimée par rapport à la dose mesurée dans l'axe du tube à $5 \mathrm{~cm}$ de l'écran). Les photons émis ont un spectre d'énergie situé 
entre 18 et $23 \mathrm{keV}$ avec un maximum autour de $20 \mathrm{keV}$. L'abdomen est situé en marge du rayonnement du tube cathodique : au cours de la grossesse, l'ombilic reste tangent ou en-dessous de l'isodose 0,008 et le débit de dose en chaque point de l'abdomen reste pratiquement le même. La figure 3 donne les variations du débit de dose reçu à la surface de l'abdomen quand on passe du pubis à l'ombilic.

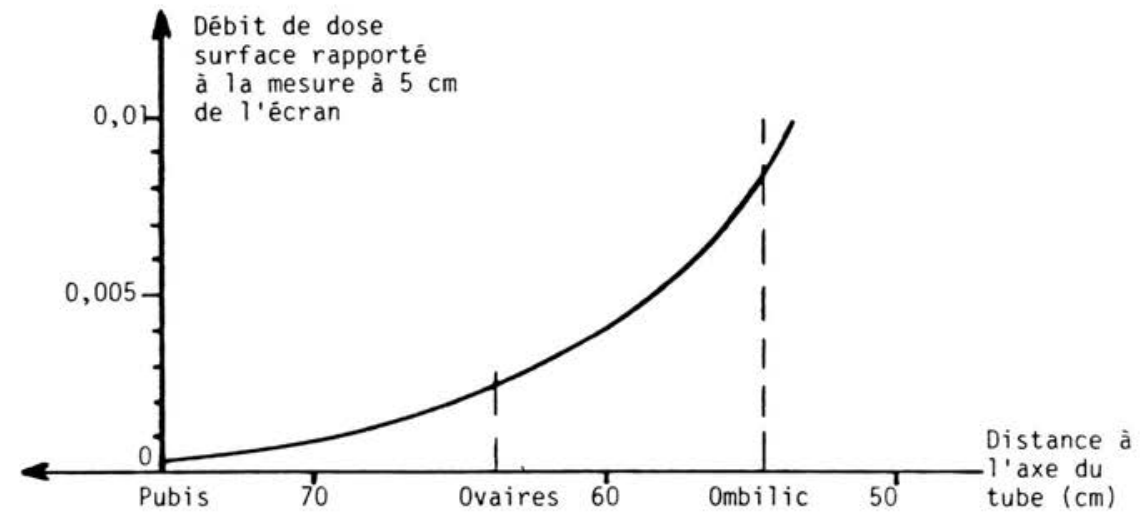

Fig. 3. - Variations du débit de dose à la surface de l'abdomen de la femme suivant la position par rapport à l'axe de l'écran.

Le fœtus bouge dans la cavité utérine, et, de ce fait, il n'est pas possible de définir un organe plus exposé qu'un autre. Pendant les deux premiers mois de la gestation qui constituent la phase embryonnaire, le niveau d'irradiation est celui reçu par les ovaires, soit $4.10^{-5}$ fois celui mesuré à $5 \mathrm{~cm}$ de l'écran qui sert de référence. Pendant la phase fœtale, à partir du $3^{\text {e }}$ mois, on prendra encore une hypothèse majorante en ne s'intéressant qu'à l'irradiation de la partie la plus exposée du fœtus. Le coefficient d'atténuation linéique des photons de $20 \mathrm{keV}$ dans les tissus mous est égal à $0,778 \mathrm{~cm}^{-1}$. Le facteur d'atténuation du rayonnement $X$ à travers la paroi abdominale et l'utérus est donc :

$$
\mathrm{r}=\mathrm{e}^{-(0,778 \times 2)}=\mathrm{e}^{-1,566}=0,211
$$

En tenant compte des courbes de croissance du fœtus et des données précédentes, le rapport du débit d'équivalent de dose au point le plus irradié au débit d'équivalent de dose mesuré à $5 \mathrm{~cm}$ de l'écran varie selon le tableau I.

TABLEAU I

Variations, jusqu'au $7^{\circ}$ mois de grossesse, du rapport du débit d'équivalent de dose au point le plus irradié du fœetus au débit d'équivalent de dose mesuré à $5 \mathrm{~cm}$ de l'écran.

\begin{tabular}{|c|c|c|c|c|c|c|c|c|}
\hline $\begin{array}{c}\text { Mois de } \\
\text { grossesse }\end{array}$ & 0 & 1 & 2 & 3 & 4 & 5 & 6 & 7 \\
\hline $\begin{array}{c}\text { Rapport des } \\
\text { débits d'équivalent } \\
\text { de dose }\end{array}$ & $4.10^{-5}$ & $4.10^{-5}$ & $4.10^{-5}$ & $2.10^{-4}$ & $10^{-3}$ & $1,2.10^{-3}$ & $1,4.10^{-3}$ & $1,6.10^{-3}$ \\
\hline
\end{tabular}




\subsection{Résultats}

Avec les hypothèses majorantes prises au paragraphe précédent et sur la base d'un débit d'équivalent de dose mesuré à $5 \mathrm{~cm}$ de l'écran de $0,2 \mu \mathrm{Sv} / \mathrm{h}(20 \mu \mathrm{rem} / \mathrm{h})$ comme on l'avait fait dans l'article précédent, l'équivalent de dose maximal reçu au point le plus irradié de l'enfant supposé immobile au cours de la gestation est de $0,121 \mu \mathrm{Sv}(12,1 \mu \mathrm{rem})$. II s'agit là d'une borne supérieure du niveau d'irradiation correspondant à un cas théorique. Nous verrons plus loin que les niveaux effectifs sont bien plus bas. Même pour ce cas pessimiste, le niveau de $0,1 \mu \mathrm{Sv}$ est l'ordre de grandeur de l'augmentation de l'irradiation naturelle au cours d'une excursion d'une demi-journée au sommet de la Tour Eiffel ou la Tour Montparnasse (dénivelé : environ $300 \mathrm{~m}$ ). C'est aussi la dose reçue au point le plus irradié par le fœtus supposé immobile, quand la mère regarde la télévision en couleurs pendant 1 mois et demi à raison de $2 \mathrm{~h}$ par jour.

\section{DISCUSSION}

On remarque, d'abord, que le cumul des doses reçues par l'enfant au cours des quatre premiers mois de gestation - période la plus radiosensible est négligeable par rapport à la dose totale reçue pendant le reste de la grossesse. Ensuite, les horaires journaliers de 6 heures correspondent aux cas particuliers de la saisie et de la restitution en ligne de données qui utilisent des consoles à tube monochrome. Les durées de travail effectives sur console polychrome sont bien plus faibles, s'agissant d'un travail partagé. Enfin, les calculs de dose ont été faits pour la partie la plus irradiée du fœtus supposé immobile. En réalité, le fœtus bouge et aucune partie du corps n'est exposée de cette façon, ce qui ramène l'irradiation maximale à un niveau 5 à 10 fois moindre.

Les calculs ont été faits pour le cas le plus défavorable d'un tube trichrome qui donne une image blanche uniforme: les canons à électrons correspondant à chaque couleur élémentaire débitent le courant cathodique maximal. Par contre, dans le cas d'une image aux couleurs saturées, les canons à électrons ne débitent pas simultanément leur courant maximum et le niveau de rayonnement descend approximativement à la moitié du niveau maximal.

Le travail sur console à traitement d'image à tube trichrome, dont le niveau d'exposition est le plus élevé, entraîne donc une irradiation moyenne de l'enfant n'excédant pas 0,01 $\mu \mathrm{Sv}(1 \mu \mathrm{rem})$. L'irradiation naturelle se situe entre 1 et $3 \mathrm{mSv}$ par an et une variation par rapport aux rayons cosmiques de $0,01 \mu \mathrm{Sv}$ correspond à une élévation en altitude de $30 \mathrm{~m}$. pendant une demi-journée ou un séjour à la montagne de 20 minutes à $1500 \mathrm{~m}$ d'altitude. Les déplacements quotidiens peuvent produire des variations de l'irradiation naturelle d'un facteur 100 fois plus élevé pendant la grossesse, et le fait de regarder la télévision couleur peut irradier 10 fois plus.

\section{CONCLUSION}

La littérature et les médias des pays anglo-saxons ont rapporté des actions juridiques accusant le rayonnement des équipements à écran ca- 
thodique d'être la cause d'avortements spontanés et de malformations congénitales $[4,5]$. Ces accusations servaient de prétexte à une certaine résistance à l'utilisation de ces nouvelles technologies. On a été jusqu'à demander à écarter les femmes enceintes de ces postes de travail ou à leur faire porter des protections individuelles. Les enquêtes qui ont suivi ont montré que ces accusations n'avaient pas de base scientifique et que les niveaux de rayonnements $X$ étaient trop bas pour observer de tels effets [1]. Ces accusations conduiraient, de la même façon, à interdire à la future mère de regarder la télévision, lui éviter tout déplacement et la confiner dans un local blindé par des écrans de plomb. Quand on mesure le gain théorique apporté, on imagine le ridicule d'une telle situation.

Même dans les cas les plus défavorables du travail devant écran trichrome, le niveau d'irradiation de l'enfant au cours de la grossesse n'est pas significatif par rapport à l'irradiation naturelle. Les niveaux sont tellement bas qu'on ne peut leur attribuer une quelconque pathologie. II en ressort qu'il n'y a donc pas lieu d'écarter les femmes enceintes des travaux sur terminal à écran cathodique.

\section{BIBLIOGRAPHIE}

[1] BOND. M.P. Statement of the American college of obstetricians and gynecologists on video display terminals and reproductive health, May 8, 1984. Washington: ACOG.

[2] COMMISSION INTERNATIONALE DE PROTECTION RADIOLOGIQUE (CIPR). Report of the task group on reference man, adopted by the Commission in October 1974. ICRP Publication 23. Oxford: Pergamon Press, 1975.

[3] DAVID G., HAEGEL P. Embryologie - Travaux pratiques - Enseignement dirigé CPEM Fascicule 1. Masson, Paris 1965.

[4] HILTS P.J. Women's groups cite VDTs in pregnancy ill clusters. The Washington Post, Saturday March 3, 1984, A9.

[5] SMITH S. Are VDT operators at risk for reproductive problems ? Obst. Gyn. News, March 15-31, 1983, p. 44.

[6] WOLBER G. Irradiation des personnes par les tubes cathodiques de télévision. Radioprotection, 1984, 19 (2) 129-144. 